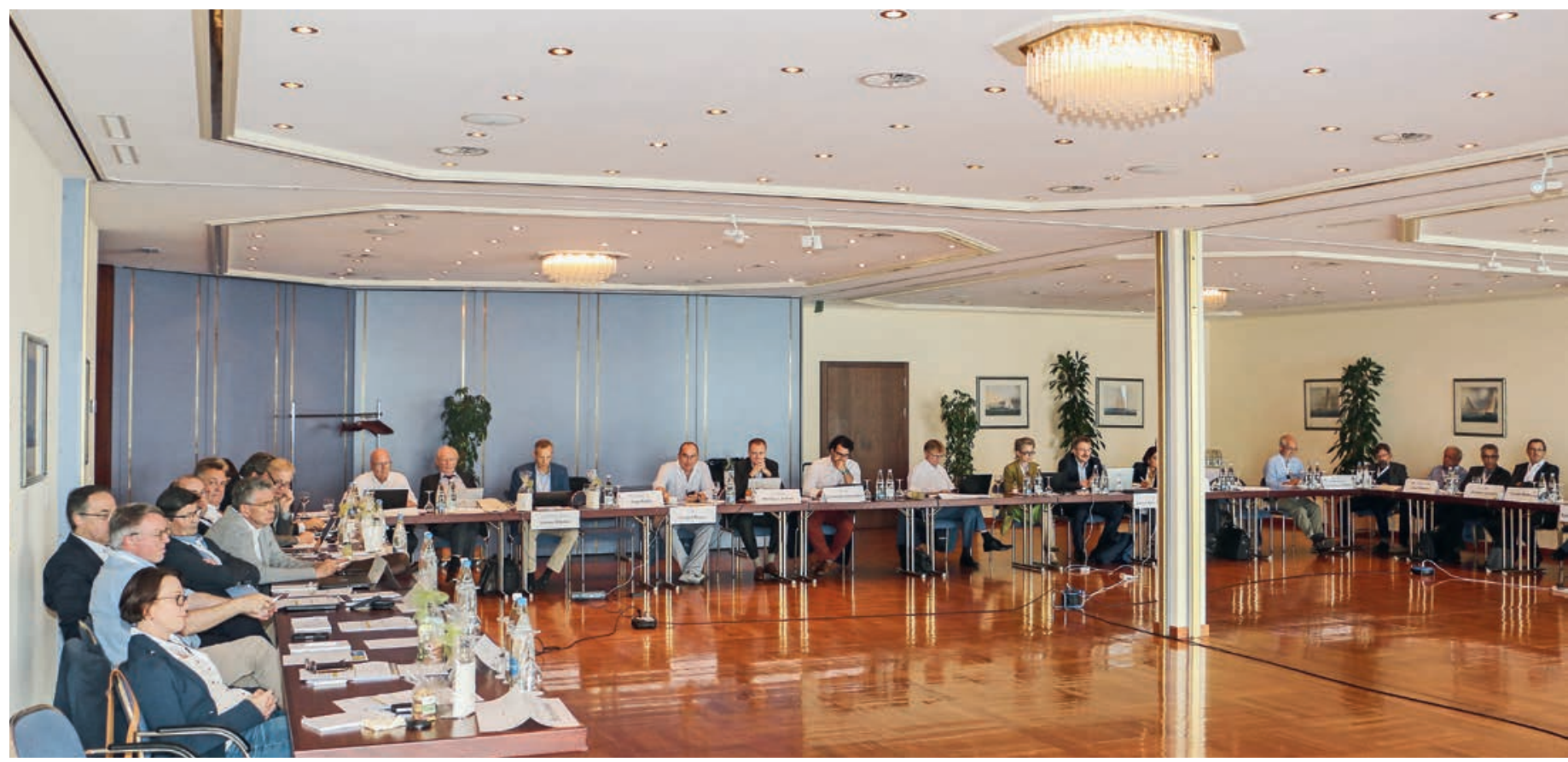

An der «Konsultativtagung Deutschsprachiger Ärztegesellschaften» 2018 nahmen Delegationen aus Deutschland, Österreich, Liechtenstein, Luxemburg, Belgien, der Schweiz und der italienischen Provinz Bozen teil.

\title{
Austausch zu drängenden Themen
}

\section{Bruno Kesseli}

Dr. med. et lic. phil., Chefredaktor

Die FMH war Gastgeberin der letztjährigen Konsultativtagung Deutschsprachiger Ärztegesellschaften, die vom 5.-7. Juli 2018 in Horn am Bodensee stattfand und von deren zweitem Tag nachfolgend berichtet wird. Alterserscheinungen waren beim Traditionsanlass auch in der 64. Auflage nicht auszumachen. Die aktuellen Herausforderungen in den Gesundheitssystemen der teilnehmenden Länder boten reichlich Stoff für einen intensiven Austausch.

Der wolkenverhangene Himmel über dem Bodensee lieferte Jürg Schlup die passende Metapher: Der Präsident der FMH zog zur Eröffnung des zweiten Symposiumstags eine Parallele zwischen der herrschenden Wetterlage und den teilweise düsteren gesundheitspolitischen Szenarien, mit denen sich die Repräsentanten der teilnehmenden Ärzteverbände in ihren Ländern auseinanderzusetzen haben. Immerhin liess Jürg Schlup, der das Vormittagsprogramm moderierte, auch etwas Optimismus in seine Metaphorik einfliessen: Es sei angekündigt, dass die Wolkendecke am Nachmittag aufreissen und ein paar Sonnenstrahlen durchlassen werde ...

\section{Medizin als arbeitsteiliger Prozess unter ärztlicher Gesamtverantwortung}

Waren tags zuvor Fragen der Aus-, Weiter- und Fortbildung im Fokus gestanden, ging es nun um die «Weiterentwicklung von Versorgungsstrukturen", mit besonderer Gewichtung von Innovation, Wettbewerb und der Sicherstellung der Versorgung. Vertreter der einzelnen Landesverbände gaben in Kurzreferaten Einblick in aktuell laufende Projekte von grösserer Tragweite. Dabei zeigte sich, dass die Herausforderungen in Deutschland, Österreich und der Schweiz in vielen Aspekten vergleichbar sind, wenngleich jedes der drei 


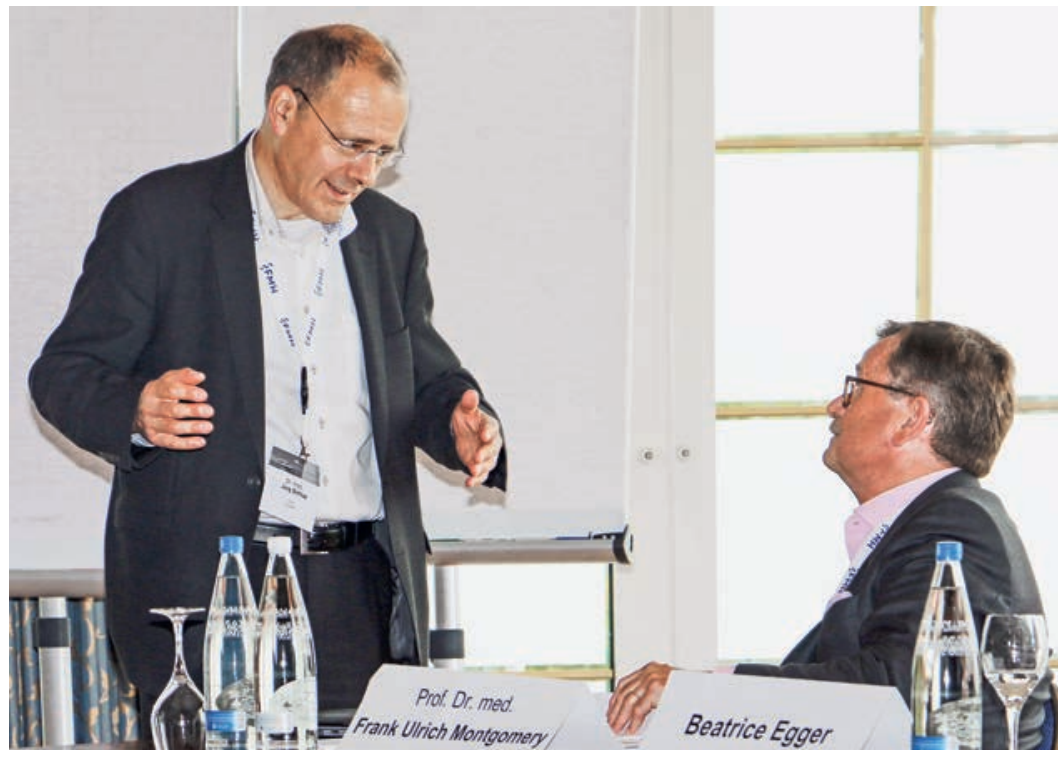

FMH-Präsident Dr. med. Jürg Schlup im Gespräch mit Prof. Dr. med. Frank Ulrich Mont gomery, Präsident der deutschen Bundesärztekammer.

Länder bezüglich der Organisation seines Gesundheitssystems Eigenheiten aufweist. Aber der Druck, die Versorgung effizienter zu gestalten, ist in allen drei Ländern vorhanden, und ökonomische Treiber spielen dabei eine zentrale Rolle.

Dr. Harald Mayer (Ö), Vizepräsident der Bundeskurie angestellter Ärzte, stellte das Konzept «Spitalsärztin/Spitalsarzt 2025» vor, mit dem sein Verband einen Beitrag zur Verbesserung der Versorgungsstrukturen im Spitalbereich leisten möchte. Das Konzept stellt die Arzt-Patienten-Beziehung und die Patientensicherheit in den Mittelpunkt und begreift die Medizin als arbeitsteiligen

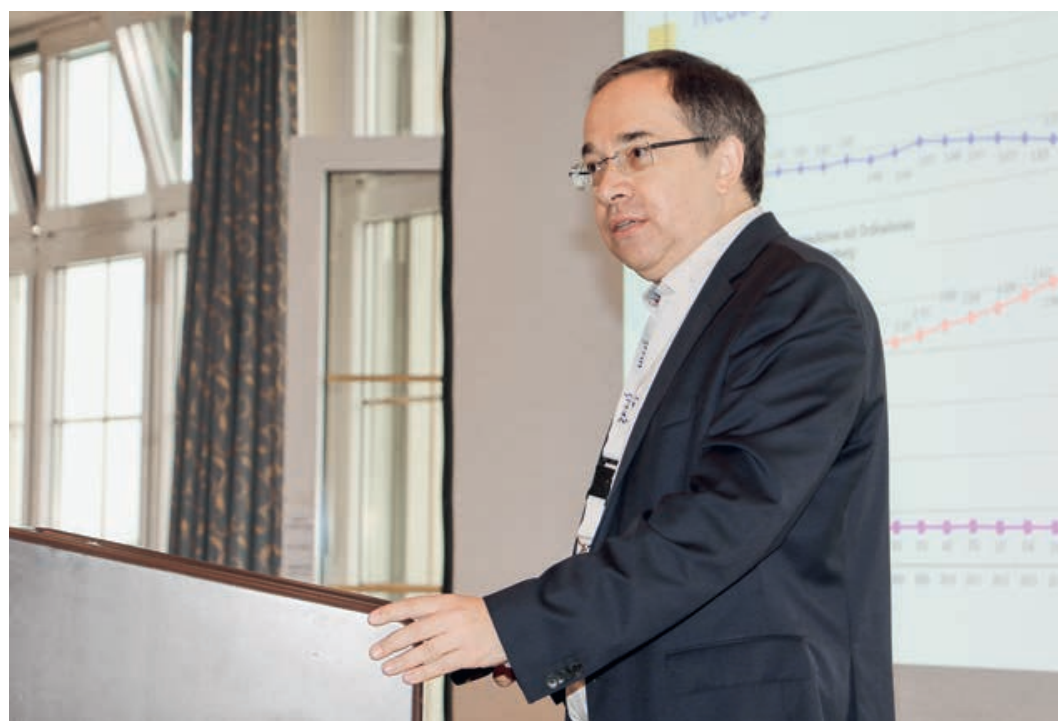

Hon.-Prof. Dr. iur. Johannes Zahrl, Kammeramtsdirektor der Österreichischen Ärztekammer, bei seinem Referat.
Prozess unter ärztlicher Gesamtverantwortung. Es sieht eine gestufte Versorgung von der ärztlichen Gruppenpraxis über stationäre Betteneinheiten und regionale Krankenhäuser bis hin zum Leit-Krankenhaus vor, das eine "24-Stunden-Vollbesetzung» aufweist. Ärztinnen und Ärzte in Ausbildung sollen in einem Rotationssystem die verschiedenen Stufen durchlaufen, und ein Fachärztepool soll eine suffiziente Versorgung auf allen Ebenen sicherstellen. Verschiedene Expertenteams werden zu medizinisch-fachlichen Einheiten zusammengefasst, die die Zusammenarbeit und Kommunikation zwischen den Spezialisten sicherstellen. Für die Ärzteschaft verspricht sich die Bundeskurie angestellter Ärzte dadurch Verbesserungen in Bezug auf die Arbeitszeiten, die Qualität der Arbeitsplätze, die Work-LifeBalance und nicht zuletzt die beruflichen Perspektiven.

\section{Trend zu hausarztgeleiteten Zentren für die Primärversorgung}

Ideen zur Reorganisation der Primärversorgung in Österreich präsentierte Hon.-Prof. Dr. iur. Johannes Zahrl, Kammeramtsdirektor der Österreichischen Ärztekammer, des österreichischen Pendants zur FMH. Bereits im Jahr 2014 wurde von der Bundes-Zielsteuerungskommission das «Konzept zur multiprofessionellen und interdisziplinären Primärversorgung in Österreich» verabschiedet. Als Eckpfeiler sieht es eine gut koordinierte Betreuung insbesondere für chronisch Kranke sowie für Kinder, Jugendliche und ältere Patienten vor. Praxen-Öffnungszeiten von 7 bis 19 Uhr sollen für kurze Wartezeiten und mehr Zeit für das Patientengespräch sorgen, so dass eine «umfassende Krankenbehandlung und Gesundheitsberatung» resultieren. Punkte im Konzept, die sich für die Ärzteschaft vorteilhaft auswirken sollen, sind beispielsweise «Teamarbeit zwischen Ärzten und weiteren Gesundheitsberufen", "geregelte Arbeitszeiten für bessere Balance zwischen Beruf und Familie» sowie eine "praxisbezogene Ausbildung und Forschung». Konkret erreicht werden soll die «neue Primärversorgung» über die Schaffung von sogenannten «Primären Versorgungseinheiten». Darunter sind ambulante Zentren zu verstehen, in denen Allgemeinmediziner/-innen in $\mathrm{Zu}$ sammenarbeit mit Fachpersonen aus Pflege, Physiotherapie, Sozialarbeit, Ernährungsberatung, Ergotherapie und anderen die Grundversorgung der Patienten einer Region sicherstellen sollen. Johannes Zahrl kritisierte unter anderem, dass die Leistungserbringer in die Erstellung der Entwürfe nicht einbezogen worden seien. Der Grundidee interprofessionell arbeitender Primärversorgungszentren könne man sich durchaus anschliessen, aber im politischen Prozess würden auch 


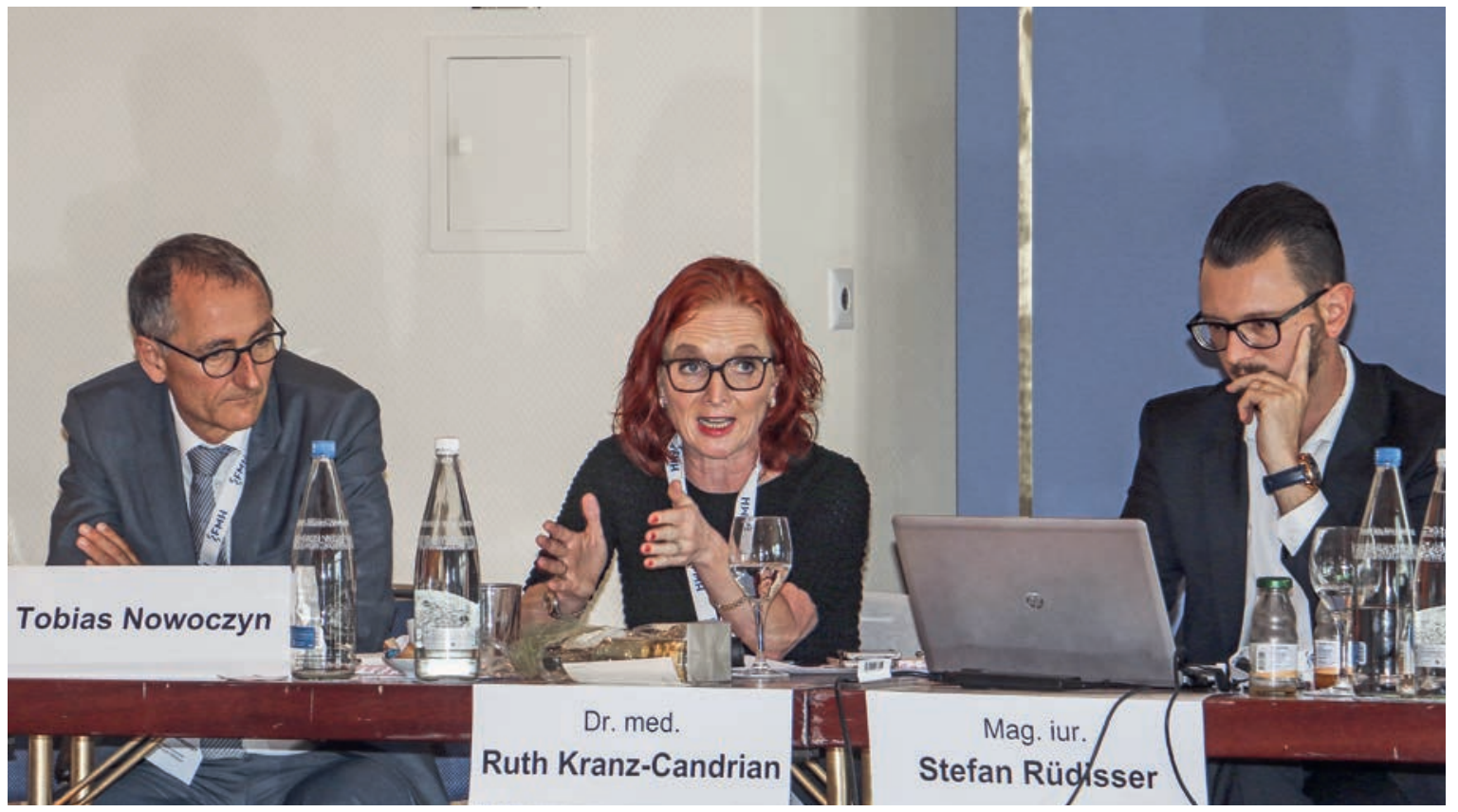

Dr. med. Ruth Kranz-Candrian, Präsidentin der Liechtensteinischen Ärztekammer, flankiert von ihrem Geschäftsführer Mag. iur. Stefan Rüdisser (rechts) und dem Hauptgeschäftsführer der deutschen Bundesärztekammer, Tobias Nowoczyn.

viele «leere Worte» gemacht. Wie die Realität dereinst aussehen wird, ist noch offen.

In eine ähnliche Richtung gehen die Vorstellungen in Deutschland, wie aus dem Referat von Dr. Max Kaplan, Vizepräsident der deutschen Bundesärztekammer (BÄK), deutlich wurde. Das Strukturkonzept der Arbeitsgruppe Allgemeinmedizin sieht eine «kollaborative Versorgung» durch "hausarztgeleitete, interprofessionelle Versorgungsteams» vor, die eine sektorenübergreifende und interprofessionelle integrierte Primärversorgung gewährleisten. Aufgewertet werden soll unter anderem der Beruf der Medizinischen Fachangestellten (MFA), des Pendants zur Medizinischen Praxisassistentin (MPA) in der Schweiz. Wie in Österreich verspricht man sich vom neuen Modell Vorteile für die Patienten wie auch für die Leistungserbringer. Auf Patientenseite werden eine effizientere, weil koordinierte Versorgung, bedarfsgerechte Öffnungszeiten der "Hausarztgeleiteten Zentren", klare Strukturen und ein grosses Leistungsangebot aus einer Hand genannt. Für die Leistungsanbieter sieht die Arbeitsgruppe attraktivere Rahmenbedingungen durch flexible Arbeitszeiten und damit eine verbesserte Vereinbarkeit von Arbeit und Familie, Entlastungsmöglichkeiten für Ärztinnen und Ärzte durch Delegation und bessere Möglichkeiten für den intra- und interprofessionellen Austausch. Auf die Frage von Jürg Schlup, ob die vorgesehenen Hausarzt-Zentren tatsächlich von Ärzten geführt würden und nicht von Managern, gab sich Kaplan sicher: «Es sind Ärzte, es müssen
Ärzte sein.» Dass administrative Aufgaben einen Grossteil der Arbeitszeit der ärztlichen Leiter beanspruchen werden - die Vermutung liegt bei 80 Prozent -, verschwieg Kaplan nicht. Er zeigte sich indessen überzeugt, dass die Versorgung sich durch solche Zentren auch in ländlichen und strukturschwachen Regionen verbessern werde.

\section{Konsultativtagung Deutschspra- chiger Ärztegesellschaften}

An der Tagung 2018 nahmen Delegationen aus Deutschland, Österreich, Liechtenstein, Luxemburg, Belgien, der Schweiz und der italienischen Provinz Bozen teil. Die FMH war Gastgeberin des Anlasses, der vom 5.-7. Juli in Horn am Bodensee stattfand. Der erste Tag unter der Leitung von Dr. Werner Bauer, Präsident des Schweizerischen Instituts für ärztliche Weiter- und Fortbildung SIWF, war Fragen der Aus- und Weiterbildung gewidmet. Am zweiten Tag fokussierten Referate und Diskussion unter der Leitung von FMH-Präsident Dr. Jürg Schlup und Prof. Frank Ulrich Montgomery, Präsident der deutschen Bundesärztekammer, auf die Themen «Weiterentwicklung von Versorgungsstrukturen und Innovation" sowie "Arztbild im Wandel. Was macht der Arzt in Zukunft bzw. was macht der Arzt der Zukunft». Der dritte Tag wurde mit einem Gastreferat eingeleitet. Prof. Dr. rer. pol. Agnes Bäker sprach zum Thema «Why the best hospitals are managed by doctors» [1]. Es folgten Länderberichte zu aktuellen, für die Ärzteschaft wichtigen Themen aus Deutschland (Prof. Frank Ulrich Montgomery), Österreich (Univ.-Prof. Thomas Szekeres), Liechtenstein (Dr. Ruth Kranz-Candrian), Luxemburg (Dr. Alain Schmit), Bozen (Dr. Monica Oberrauch), Belgien (Dr. Michael Heinen) und der Schweiz (Dr. Jürg Schlup). 


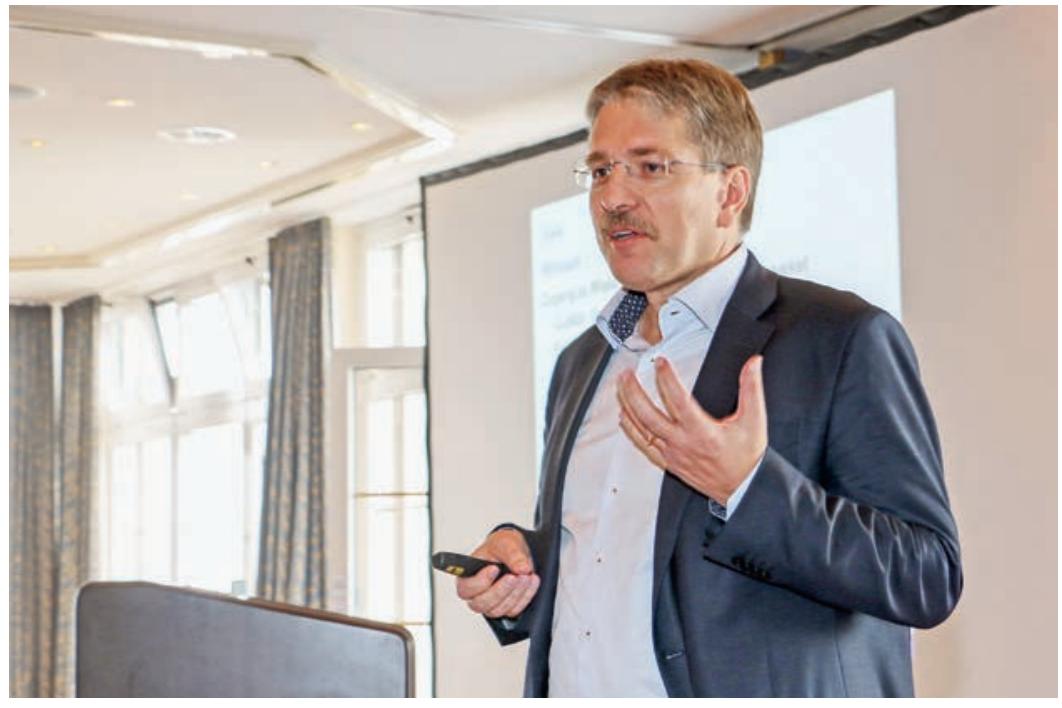

"Der Patient auf Augenhöhe» war Thema des Referats von Dr. med. Carlos Quinto, Mitglied des Zentralvorstands der FMH.

\section{Bedeutung der interprofessionellen Zusammenarbeit unbestritten}

Wie die mehrfach als zukunftsweisend angesprochene interprofessionelle Zusammenarbeit funktionieren kann, zeigte Barbara Weil, Leiterin der Abteilung Public Health und Gesundheitsberufe der FMH, am Beispiel der Prävention. Dass der Prävention in der Gesundheitsversorgung einer Bevölkerung ein hoher Stellenwert zukommen sollte, ist breit anerkannt. Die praktizierende Ärzteschaft, so Barbara Weil, sehe sich aber mit unterschiedlichen Aktivitäten und Erwartungen konfrontiert. Koordination, ein gemeinsames Verständnis von Qualität und gemeinsame Rahmenbedingungen seien vor diesem Hintergrund besonders wichtig. Dank interprofessioneller, koordinierter $\mathrm{Zu}$ sammenarbeit wurde in der Schweiz bereits eine Reihe von Hilfsmitteln entwickelt, die eine umfassende, systematische und nachweislich wirksame Prävention und Gesundheitsförderung begünstigen. Zu nennen sind in diesem Zusammenhang etwa «Eviprep», eine generische «Evidenz-Datenbank» (www.eviprep.ch), Motivational Interviewing als generische Kommunikationsform, spezifische Vertiefungsmodule zu Risikofaktoren, Gesundheitscoaching als Beratungskonzept oder Shared Decision Making und Planning tools als weitere Optionen.

\section{Den Wandel selbst gestalten}

Dass die interprofessionelle Zusammenarbeit künftig noch an Bedeutung gewinnen wird - und dies zu Recht -, wurde in der Diskussion zu den Referaten des Vormittags von mehreren Votanten unterstrichen.
Dr. Günther Jonitz, der später selbst als Referent auftrat, bezeichnete sie als Grundprinzip. Was die Organisation der Primärversorgung betrifft, zeigte er sich überzeugt, dass freiberuflich tätige Ärztinnen und Ärzte am effizientesten arbeiten. Dagegen wurde argumentiert, die heutige Ärztegeneration sei kaum mehr bereit, (finanzielle) Risiken einzugehen, und ziehe deshalb eine Anstellung vor. Gemäss dem Präsidenten der deutschen Bundesärztekammer, Prof. Frank Ulrich Montgomery, sind indes auch Modelle mit dosiertem Risiko vorgesehen. Ärztinnen und Ärzte könnten in «Schnupperpraxen» während dreier bis fünf Jahren risikofrei Erfahrungen sammeln. Sollten sie an dieser Form der Primärversorgung Gefallen finden, hätten sie in einem zweiten Schritt die Möglichkeit, die Praxis zu übernehmen.

Mit den «Herausforderungen an den Arztberuf» setzte sich zum Abschluss des Vormittagsteils der bereits genannte Günther Jonitz, Präsident der Ärztekammer Berlin, auseinander. An einer Vielzahl von schlagenden Beispielen und durchaus humorvoll zeigte er auf, wie sich die ärztliche Rolle in der jüngeren Vergangenheit gewandelt hat. Ärztinnen und Ärzte sind von «Alleinverantwortlichen» zu "Letztverantwortlichen" geworden, und «Selbstbestimmung» wurde zunehmend durch «Fremdbestimmung» abgelöst. Seine Ausführungen erschöpften sich aber keineswegs darin, Missstände zu beklagen und vergangenen Zeiten nachzutrauern - ganz im Gegenteil. Jonitz sieht die adäquate Antwort in einer verstärkten Professionalisierung des Ärztestands. Nun gehe es darum, Verantwortung zu übernehmen und den Wandel selbst zu gestalten. Die ärztlichen Kompetenzen müssten gestärkt und die Qualifikationen erweitert werden. Letztlich habe sich die Ärzteschaft zu entscheiden, ob sie «Koch» oder «Kellner» sein wolle. «Die gesundheitspolitische Diskussion», schloss er seine Ausführungen, "gewinnt der, der glaubhaft Anwalt der Patientenversorgung ist.»

\section{Arztbild im Wandel}

Verschiedene der von Günther Jonitz angesprochenen «Herausforderungen» wurden von der Referentin und den Referenten des Nachmittags aufgegriffen. Um den «Patienten auf Augenhöhe» drehte sich das Referat von FMH-Zentralvorstandsmitglied Dr. Carlos Quinto. Er identifizierte Shared Decision Making und Empowerment als wichtige Elemente einer patientenzentrierten medizinischen Versorgung im Sinn der Ottawa-Charta zur Gesundheitsförderung. Seine Ausführungen machten aber auch deutlich, dass eine Asymmetrie in der Arzt-Patienten-Beziehung nicht wegdiskutiert werden kann, gerade wenn man die Patienten ernst 


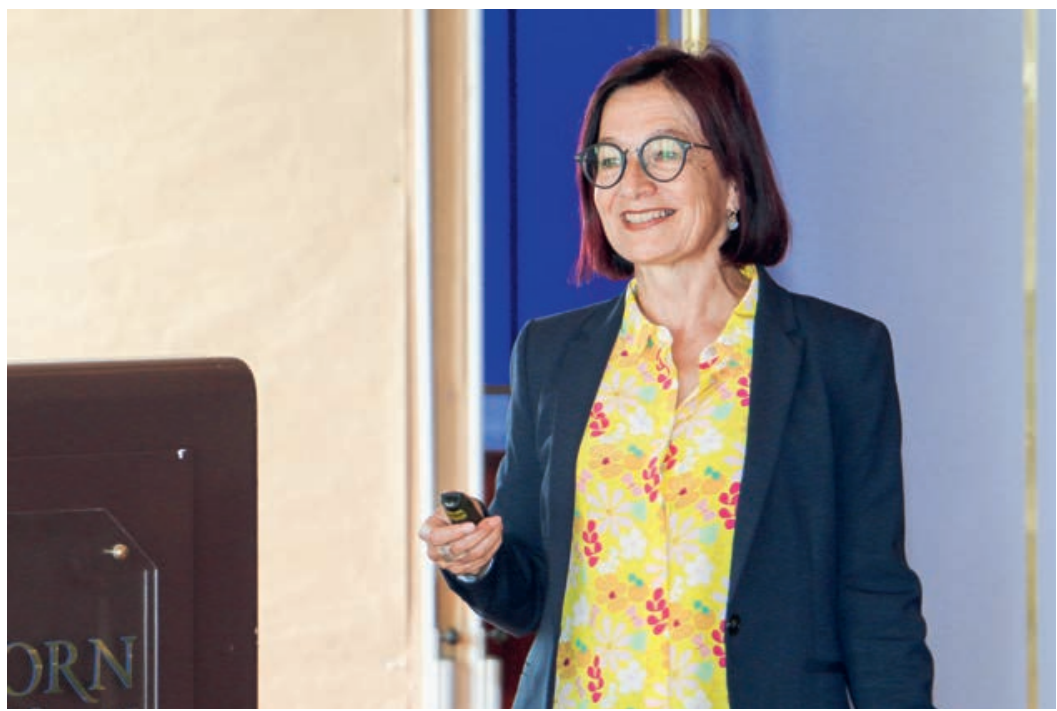

Dr. med. Yvonne Gilli, Mitglied des Zentralvorstands der FMH, sprach zum Thema Digitalisierung.

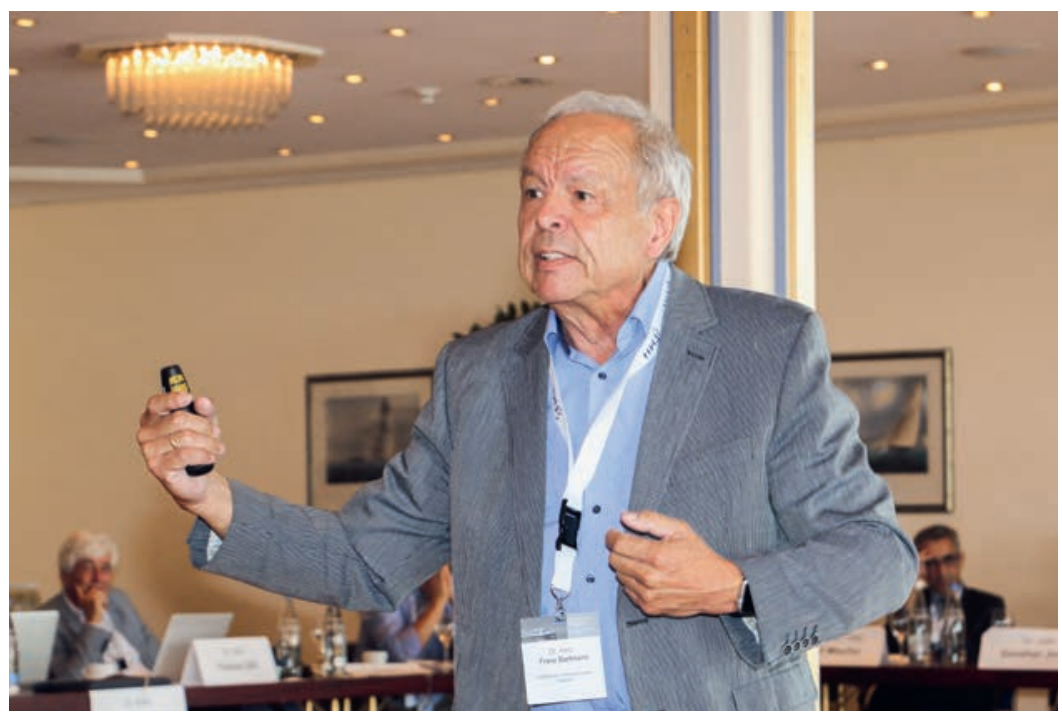

Dr. med. Franz Bartmann, Präsident der Ärztekammer Schleswig-Holstein, befasste sich in seinem temperamentvollen Referat mit «neuen Versorgungsstrukturen infolge Digitalisierung».

* EMR = Electronic Medical Record.

Korrespondenz: bkesseli[at]emh.ch nimmt. Tragfähige gemeinsam von Patient und Arzt getroffene Entscheidungen sollten diesem Umstand Rechnung tragen.

PD Dr. Jörg Hutter, Vizepräsident der Ärztekammer Salzburg, setzte sich unter dem Titel «Bürokratisierung, Codierung, Verwaltung und Qualitätsmanagement: Wo bleibt die Zeit für ärztliche Tätigkeit?» mit einem Thema auseinander, das auch in der Schweiz und in Deutschland intensiv diskutiert wird. EMR*-
Systeme versprechen zwar Effizienzgewinn und eine Verbesserung der Behandlungsqualität und -sicherheit für die Patienten, führen aber nicht per se zu einer Entlastung des ärztlichen Personals von administrativen Leistungen. Neben der Delegation von Administrativarbeiten an andere, teilweise sogar auf die medizinische Dokumentation spezialisierte Berufsgruppen muss gemäss Hutter auch die Ausbildung der Assistenzärztinnen und -ärzte den neuen digitalen Möglichkeiten angepasst werden. Inwieweit die verschiedenen diskutierten Massnahmen die Situation verbessern können, lässt sich noch kaum abschätzen.

\section{Smartphone als Stethoskop des 21. Jahrhunderts?}

Der Abschluss des Nachmittags war dem Einfluss der Digitalisierung auf die Arztrolle und die Arzt-Patienten-Beziehung gewidmet. Bestritten wurde dieser Teil von FMH-Zentralvorstandsmitglied Dr. Yvonne Gilli sowie Dr. Josef Mischo, Präsident der Ärztekammer Saarland, und Dr. Franz Bartmann, Präsident der Ärztekammer Schleswig-Holstein. Die Referenten waren sich einig, dass nicht die Digitalisierung «das Problem» sei, sondern deren Umsetzung. Yvonne Gilli wies darauf hin, dass beispielsweise das Gefälle zwischen armen und reichen Regionen auf der Welt durch die Digitalisierung nicht ausgeglichen werde und die Digitalisierung per se die Produktivität nicht erhöhe. Franz Bartmann konstatierte, dass die Medizin in Bezug auf die Ausschöpfung der digitalen Möglichkeiten anderen Bereichen - etwa der Musikindustrie - noch hinterherhinke. Er zeigte sich überzeugt, dass das Smartphone dank telemedizinischer Fortschritte zum «Stethoskop des 21. Jahrhunderts" werde. Seiner Prognose, dass Apps zwar Ärzte nicht ersetzen werden, dass aber die Digitalisierung das ärztliche Rollenbild grundlegend verändern wird, konnten sich wohl die meisten Teilnehmenden anschliessen.

\section{Bildnachweis}

Fotos Bruno Kesseli

\section{Referenz}

1 Siehe zu diesem Thema auch das online zugängliche Interview in der Schweizerischen Ärztezeitung vom 8. November 2017: Kesseli B. «Evidenz spricht für Besetzung von Führungspositionen mit Ärzten». Interview mit Agnes Bäker, Assistenzprofessorin für Management von Nonprofit-Organisationen an der Universität Zürich. Schweiz Ärzteztg. 2017;98(45):1487-9. DOI: https://doi.org/10.4414/saez.2017.06129 